\title{
STEM Imaging and Analysis of Ferritin Nanoparticles in Organs: Spatial and Temporal Association of Ferritin with Invader Nanoparticles and Oxidation States Revealed
}

\author{
Uschi M. Graham ${ }^{1,2}$, Alan K. Dozier ${ }^{2}$, Chen Wang ${ }^{2}$, Joseph E. Fernback ${ }^{2}$, M. Eileen Birch ${ }^{2}$, Guenter \\ Oberdoerster $^{3}$, and Burtron H. Davis ${ }^{1}$ \\ 1. Center for Applied Energy Research, University of Kentucky, Lexington, KY, 40511, USA \\ 2. Division of Applied Research and Technology, National Institute for Occupational Safety and Health, \\ Cincinnati, OH, 45226, USA \\ 3. School of Medicine and Dentistry, University of Rochester, Rochester, NY, 14642, USA
}

High ferritin serum blood levels have long been associated with inflammation and disease and typically reference the ferritin protein with little or no iron content [1]. High serum ferritin represents a commonality in inflammatory disease and can be investigated by scanning transmission electron microscopy (STEM), energy dispersive spectroscopy (EDS), and electron energy loss spectroscopy (EELS). Because ferritin nanoparticles produce such a weak EDS signal, we relied on EELS for identification of ferritin iron. We utilized STEM imaging of organ tissues to examine the bio-mineralized iron portion located within the ferritin protein shell, and to demonstrate a spatial and temporal association with various invader nanoparticles (ceria and amorphous silica) after either inhalation or intravenous exposure and subsequent sequestration inside organs (lung, liver, spleen and brain were examined). Surprisingly, STEM imaging reveals that different invader nanoparticles, even after being delivered to organ tissues via different uptake routes (inhalation, intravenous), show a common response, specifically, the placement of ferritin iron nanoparticles in the immediate vicinity of the invader nanoparticles. STEM reveals abundant ferritin in local environments surrounding individual and agglomerated invader nanoparticles, and this close proximity and spatial arrangement has not been described previously. EELS confirms the iron content of the nanoparticles. Ferritin nanoparticles are typically present inside cells, and outside to some lesser extent, but not at the high concentrations observed in this study. The mechanisms that control the abundant in vivo response of ferritin nanoparticles may relate to an inflammatory response caused by intruder nanoparticles, triggering an upregulation of iron that explains the ferritin-rich areas observed in STEM images. Size ranges, oxidation states and cellular associations of the bio-mineralized ferritin are illustrated with high resolution STEM images, EDS, and EELS in the vicinity of invader nanoparticles.

In Figure 1, the images were acquired for rat lung tissue from an inhalation study on amorphous $\mathrm{SiO}_{2}$ nanoparticles. The top row shows a TEM image (a) and a diagram (b) illustrating the ferritin localization around the $\mathrm{SiO}_{2}$ agglomerate. Below that are a series of STEM images (c, d, e) zooming in on the ferritin. At the lower right, an EELS spectrum localized on a ferritin particle shows its iron content (f). The ferritin ranges in size from $7 \mathrm{~nm}$ to $11 \mathrm{~nm}$ as shown in the lower left STEM image. Figure 2 images were taken of spleen tissue from a rat that had intravenous $\mathrm{CeO}_{2}$ exposure. Agglomerated $\mathrm{CeO}_{2}$ is seen surrounding a macrophage subcellular structure in the upper left image (a). Within the structure are abundant ferritin nanoparticles $(b, c)$. The final image in the lower right shows a Fe LII-III EELS spectrum for one of the nanoparticles, revealing its iron content (d).

This work was performed using the NIOSH JEOL 2100F field emission TEM/STEM with an Oxford EDS system and a Gatan Tridiem EELS spectrometer located in Cincinnati, OH. STEM imaging and EELS analysis were done using a $0.2 \mathrm{~nm}$ probe. 


\section{References}

[1] D.B. Kell, E. Pretorius, Metallomics, 6 (2014), p. 748-773.

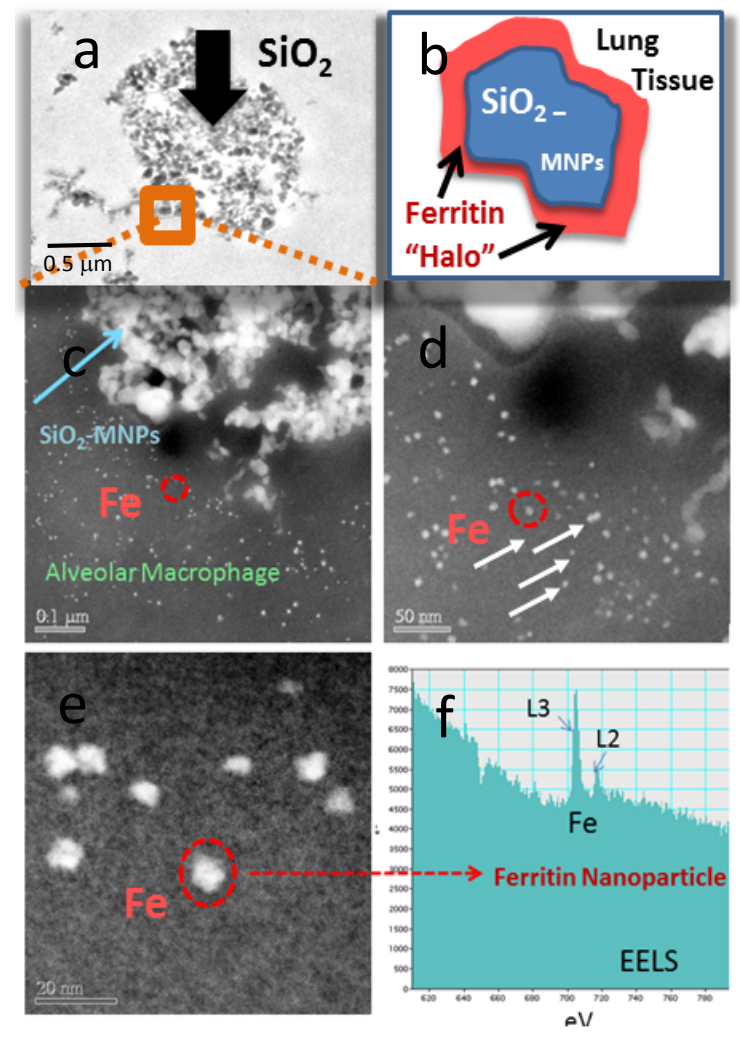

Figure 1

$\mathrm{SiO}_{2}$-nanoparticles in TEM (a) and dark field STEM images (c, d, e) showing cellular structure of the alveolar macrophage. The $\mathrm{SiO}_{2}$-MNPs are surrounded by ferritin nanoparticle halos (diagram in b) identified using EELS spot analysis (f).

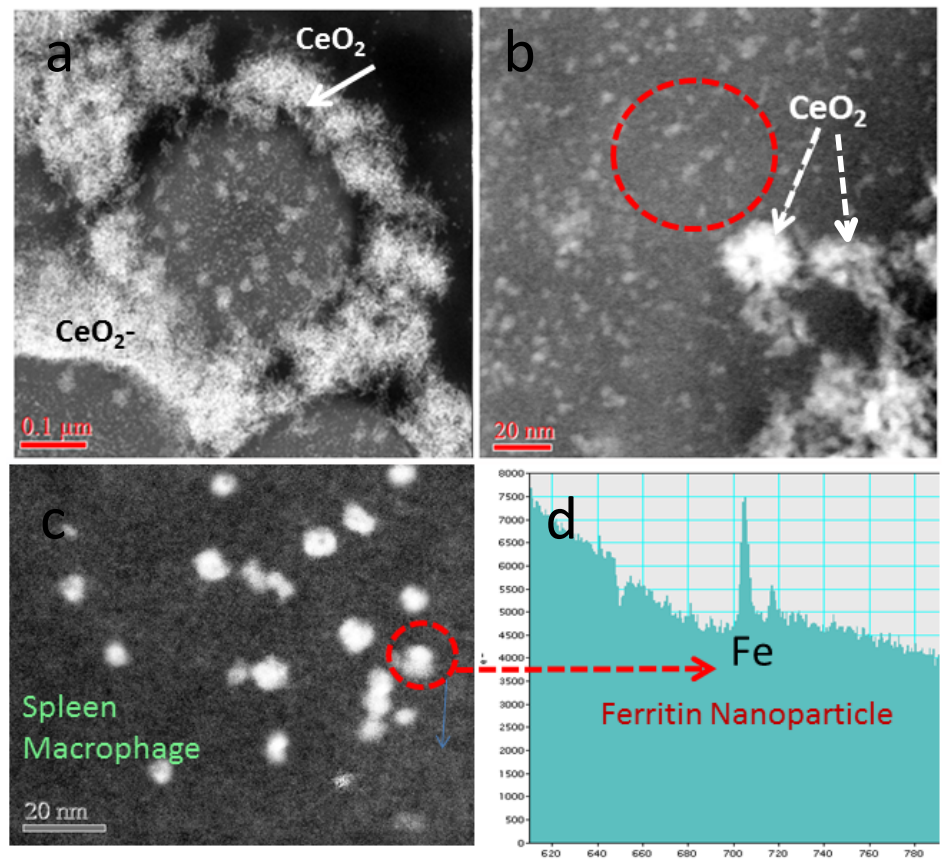

\section{Figure 2}

$\mathrm{CeO}_{2}$-nanoparticles in dark field STEM showing cellular structure in the spleen macrophage (a). The $\mathrm{CeO}_{2}-\mathrm{MNPs}$ localize around cellular inclusions and are surrounded by ferritin nanoparticles (b) identified using EELS spot analysis (c, d). 